\title{
Marker-trait associations for barley grain quality traits identified in Karaganda and Kostanay regions using GWAS
}

Genievskaya Y. ${ }^{1,2 *}$, Almerekova S. ${ }^{1}$, Abugalieva A. ${ }^{3}$, Abugalieva S. ${ }^{1}$, Turuspekov Y. ${ }^{1}$

${ }^{1}$ Institute of Plant Biology and Biotechnology, Almaty, Kazakhstan

${ }^{2}$ Al-Farabi Kazakh National University, Almaty, Kazakhstan

${ }^{3}$ Kazakh Research Institute of Agriculture and Plant Industry, Almalybak, Kazakhstan

*email: julia.genievskaya@gmail.com

Barley (Hordeum vulgare L.) is the second important cereal crop after wheat in Kazakhstan. Barley production in the country is oriented for livestock feed and brewing. Generally, the requirements for barley grain quality used in brewing and livestock feeding are different, and, usually, the good quality of the grain is associated with a lower yield. Modern genomic technologies help to identify important quantitative trait loci and determine valuable crop genotypes for breeding. Spring barley collection, including 557 USA's accessions and 104 accessions from Kazakhstan, was studied for major grain quality traits of barley grown in Karaganda (center) and Kostanay (north) regions in 2010 and 2011. The collection was previously genotyped using barley iSelect 9K SNP array resulted in 2,344 polymorphic markers. Phenotypic and genotypic data were used for the searching of marker-trait associations using GWAS. As a result, 69 marker-trait associations $(P<0.001)$ were identified for 10 grain quality traits: 13 for grain protein content, 9 for glutenin content, 9 for grain plumpness, 8 for starch content, 7 for amylose content, 7 for grain extractivity, 6 for grain glume, 5 for hordein content, 3 for albumin, and 2 for grain $\beta$-glucan content. Sixty-nine marker-trait associations were unified in 27 QTLs, considering the pleiotropic effect of several markers. Seven of these pleiotropic QTLs were previously described as associated with barley yield-related traits. Both negative and positive effects were observed for all studied traits; therefore, QTLs identified in the study may be used for the breeding of barley for different purposes and with different quality of the grain. QTLs detected in different regions of Kazakhstan have a potential usage for local barley breeding programs.

Financial support: grant AP08052804 "Development and validation of KASP arrays efficiency for key productivity and grain quality traits in two-rowed spring barley" (MES $\mathrm{RK})$. 